\title{
Influencia de la composición y las condiciones de procesado en la resistencia a la corrosión de materiales compuestos base aluminio
}

\author{
A. JIMÉNEZ-MORALES, E. M. RUIZ-NAVAS, J. B. FOGAGNOLO, J. M. TORRALBA \\ Dpto. Ciencia de Mat. e Ing. Metalúrgica. Universidad Carlos III de Madrid.
}

\begin{abstract}
Este trabajo trata de analizar el comportamiento frente a la corrosión de una serie de materiales compuestos, así como de estudiar la influencia del refuerzo y el tipo de molienda con respecto a la aleación base sin reforzar. Para ello, se ha fabricado una serie de materiales compuestos con matriz de aluminio, aleación 6061, reforzados con un $5 \%$ en peso de $\mathrm{Si}_{3} \mathrm{~N}_{4^{\prime}} \mathrm{ZrB}_{2}$ y AlN, empleando como técnicas de mezcla y homogeneización dos tipos de molienda, de baja y alta energía, respectivamente. El trabajo se ha basado principalmente en el estudio de la evolución con el tiempo del potencial de corrosión, del potencial de picadura y de la zona pasiva de cada material por técnicas electroquímicas de corriente continua. Los resultados más significativos revelan la influencia tanto del refuerzo como del empleo de las distintas técnicas de mezcla, tanto en el potencial de corrosión como en el de picadura.
\end{abstract}

Palabras clave: Materiales Compuestos de Matriz Metálica, Aluminio, Corrosión, Molienda de Alta Energía, Potenciometría.

\section{Influence of the composition and processing conditions on the corrosion resistance of aluminium-based composite materials}

This work tries to analyze the corrosion behavior of a serie of composite materials, as well as to study the influence of the reinforcement and milling technique on the unreinforced base alloy corrosion resistance. For this purpose composite materials, base 6061 aluminum alloy and reinforced with $\mathrm{Si}_{3} \mathrm{~N}_{4}, \mathrm{ZrB}_{2}$ and AlN (5\% wt.) have been manufactured, by means of both low and high energy milling techniques for the blending step. The study is mainly focused on the evolution of the corrosion potential versus time, pitting potential as well as the pasive zone of each material by means of continuous current electrochemical techniques. The most significant results reveal the influence of the reinforcement as well as the employment of different blending techniques, both on the corrosion and pitting potential.

Keywords: Metal Matrix Composites, Aluminium, Corrosion, High-Energy Milling, Potenciometric curve.

\section{INTRODUCCIÓN}

Los materiales compuestos de matriz metálica (MMCs) reforzados con partículas constituyen una clase de materiales de nueva generación, cuyas propiedades suelen ser diseñadas a medida para adaptarse a una aplicación determinada. Dentro de ellos, los materiales compuestos de matriz de aluminio (1), gracias a la excelente combinación de propiedades que presentan, han tenido una fuerte evolución en los últimos años, presentando numerosas aplicaciones industriales, principalmente en el sector de la automoción. Las principales ventajas que presentan estos materiales se basan en su ligereza, típica de las aleaciones ligeras, el fácil procesamiento y el incremento de propiedades que proporciona el refuerzo, merced a la adición de partículas duras que aumentan la dureza de las aleaciones base. La selección de la matriz juega un papel muy significativo a la hora de determinar las propiedades finales del compuesto. En particular, la ductilidad y la tenacidad a fractura están fuertemente influenciadas por ella y por su posterior tratamiento térmico. Además, las propiedades que presenta el material compuesto final a elevadas temperaturas o a corrosión dependen directamente de las características de la matriz. Así las matrices más comunes son las de la serie 2xxx por su excelente combinación de propiedades y las de la serie $6 x x x$ por su elevada resistencia a corrosión en ambientes severos y facilidad de procesado $(2,3,4)$.

Por otro lado, el proceso pulvimetalúrgico presenta varias ventajas en comparación a los métodos convencionales de fabricación, como son el moldeo o las técnicas de deformación. El control de la for- ma y dimensiones de la pieza con un mínimo de pérdida de material y el aumento del uso de materia prima de bajo coste viene favoreciendo el desarrollo de estas técnicas que se emplean ya ampliamente para materiales compuestos de matriz metálica, y en especial para aleaciones base aluminio. El proceso de obtención de materiales compuestos de matriz de aluminio siguiendo el proceso pulvimetalúrgico consiste básicamente en la mezcla de los polvos metálicos que constituyen la matriz y el refuerzo, en estado sólido, seguida de la etapa de compactación y sinterización. Sin embargo, las propiedades mecánicas de estos materiales compuestos se pueden mejorar si la etapa de mezcla y homogeneización se realiza a través de técnicas de molienda de alta energía, así como si la etapa de sinterización se sustituye por un proceso de consolidación termomecánico a alta temperatura, principalmente de extrusión. Esta técnica proporciona materiales de elevada calidad, con relación a la correcta distribución del refuerzo (mayor homogeneidad) y a la densificación casi total (ausencia de porosidad), y ha sido validada para la fabricación de este tipo de materiales $(5,6,7)$.

Por otro lado, las propiedades a corrosión de los MMCs no pueden ser mejoradas, en principio, debido a la presencia del refuerzo (8). El mayor problema que se plantea en estos materiales es el estudio de los efectos negativos relativos a la incorporación de estos. El empleo de moliendas de baja energía en la etapa de mezcla del refuerzo conlleva mejoras en la distribución del refuerzo que repercuten en las propiedades mecánicas, sin que el comportamiento frente a corrosión se 
vea modificado con respecto a la matriz. Sin embargo, la molienda de alta energía provoca modificaciones en las características de la matriz, introduciendo un nivel de deformación y defectos que repercute no sólo directamente en la mejora de propiedades mecánicas de material compuesto final, sino que indirectamente pueden influir positivamente en el comportamiento frente a corrosión.

\section{PROCEDIMIENTO EXPERIMENTAL}

Para la fabricación de los distintos materiales compuestos se han empleado polvos prealeados $(75 \mu \mathrm{m})$ de la aleación de aluminio 6061 $(0.96 \mathrm{Mg}, 0.69 \mathrm{Si}, 0.24 \mathrm{Cr}, 0.19 \mathrm{Cu}, 0.06 \mathrm{Fe})$ para la matriz y como refuerzos un 5 y $15 \%$ en peso de $\mathrm{AlN}, \mathrm{Si}_{3} \mathrm{~N}_{4}$ y $\mathrm{ZrB}_{2}$. Un grupo de materiales compuestos se ha fabricado a partir de los polvos de la aleación de aluminio y cada uno de los refuerzos, mezclados en primer lugar en un molino de bolas convencional de baja energía (LE) durante 1.5 horas a 150 r.p.m. El otro grupo de materiales se fabricó mediante molienda de alta energía (HE) en un molino de bolas escéntrico (Fritshc Gmbh, Pulverisette 6) durante 10 h. a 700r.p.m., con relación bolas/ carga 6 a 1 y con $1 \%$ en peso de Microwax C (Hoechst) como agente controlador de proceso (PCA).

Una vez realizada la etapa de mezcla y homogeneización, los distintos polvos se compactaron uniaxialmente a $300 \mathrm{MPa}$ tras lo cual se llevó a cabo la etapa de consolidación en caliente mediante la extrusión, a $500^{\circ} \mathrm{C}$, con una relación de extrusión de 25:1 y con una velocidad de extrusión de $30 \mathrm{~cm} / \mathrm{min}$.

Para el estudio del potencial de corrosión del material consolidado se realizaron ensayos de inmersión según la norma ASTM G110 (inmersión durante 6 horas de las probetas en una disolución acuosa de $57 \mathrm{~g}$ de $\mathrm{NaCl}$ y $10 \mathrm{ml} \mathrm{H}_{2} \mathrm{O}_{2}$ en un litro de agua). También se realizaron ensayos de inmersión en una disolución acuosa 3,5\% de $\mathrm{NaCl}$, haciendo medidas del potencial de corrosión a lo largo del tiempo hasta un máximo de siete días de ensayo. El área de exposición fue de $177 \mathrm{~mm}^{2}$.

El estudio del potencial de picadura y repasivación se realizó a través de curvas de polarización, empleando la celda electrolítica EG\&G Flat Cell, con un contraelectrodo de platino. La disolución para estos ensayos fue una disolución acuosa de $3.5 \%$ de $\mathrm{NaCl}$, previamente desaireada. Los datos se registraron en un potenciostato Perkin Elmer modelo 263-1, empleando como electrodo de referencia un electrodo estándar de calomelanos saturados (SCE).

\section{RESULTADOS Y ANÁLISIS}

\subsection{Estudio del potencial de corrosión}

Los resultados obtenidos en relación al potencial de corrosión de los materiales compuestos reforzados con $\mathrm{Si}_{3} \mathrm{~N}_{4}$ y Z ZrB ${ }_{2^{\prime}}$ así como el de la aleación base sin reforzar, obtenidos a partir del ensayo de inmersión normalizado, aparecen representados en la figura 1.

En ella se puede observar, en primer lugar, una clara tendencia en todos los materiales a la estabilización del potencial en el tiempo. El valor de potencial de corrosión obtenido para la aleación base AA6061 sin reforzar fue de $-765 \mathrm{mV}$, concordando con resultados obtenidos por otros autores (9). Para los materiales compuestos este valor se encuentra entre -756 y $-784 \mathrm{mV}$.

El análisis de dicha figura permite observar que, a igualdad de contenido en refuerzo y método de molienda, los valores de potencial de corrosión para los materiales reforzados con $\mathrm{Si}_{3} \mathrm{~N}_{4}$ son mayores que los obtenidos para los materiales reforzados con $\mathrm{ZrB}_{2}$. Esta tendencia

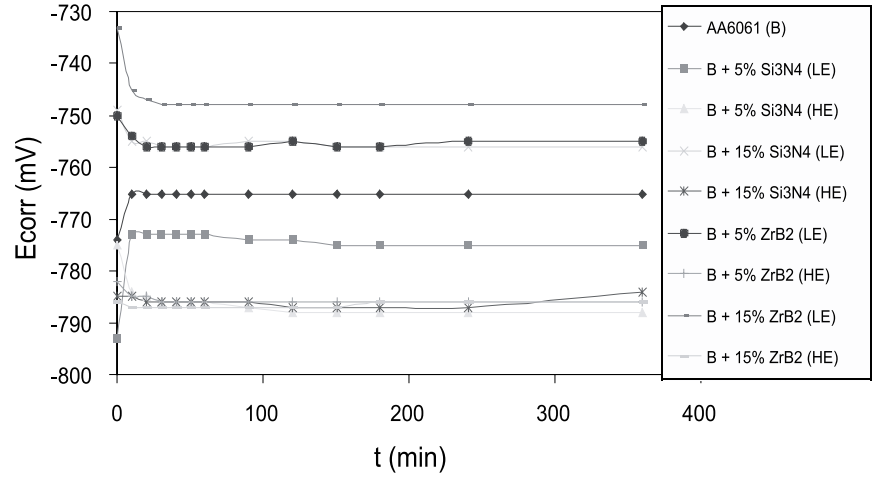

Figura 1.- Potencial de corrosión de la aleación AA6061 y de los materiales compuestos. Ensayo de inmersión normalizado (ASTM G110).

puede indicar que, en aquellos materiales compuestos cuya matriz está constituida por la misma aleación de aluminio, el tipo de refuerzo influye en el valor del potencial de corrosión obtenido. Considerando que cuanto menor sea el valor del potencial de corrosión más anódico es el comportamiento del material ensayado, se puede concluir que los materiales con refuerzo de $\mathrm{Si}_{3} \mathrm{~N}_{4}$ han presentado un mejor comportamiento frente a la corrosión electroquímica que los materiales reforzados con $\mathrm{ZrB}_{2}$.

Por otro lado, los materiales obtenidos a partir de molienda de alta energía (HE), presentan potenciales de corrosión muy similares, independientemente de la cantidad de refuerzo añadido a la matriz. De igual forma, se puede observar que el valor de potencial de corrosión de los materiales fabricados en procesos de baja energía (LE) es siempre menor que el potencial obtenido para los materiales conformados por alta energía, a igual porcentaje de refuerzo. Al igual que en caso anterior, esta tendencia probablemente indica que la técnica de molienda empleada en la etapa de mezcla y homogeneización puede influir más en el comportamiento frente a la corrosión que el contenido en refuerzo. Además, aparentemente, los materiales obtenidos por molienda de alta energía presentan mayor susceptibilidad al ataque por corrosión que los materiales fabricados por baja energía.

Si se analizan los resultados obtenidos a partir del ensayo de inmersión no normalizado, y representados en la figura 2, se puede observar que el valor de potencial obtenido para la aleación base AA6061 es mayor que el alcanzado por los materiales compuestos, excepto en el caso del material reforzado con un $5 \%$ de $\mathrm{Si}_{3} \mathrm{~N}_{4}$ y obtenido por molienda de alta energía.

Por otro lado, los materiales reforzados con $\mathrm{AlN}$ y $\mathrm{ZrB}_{2}$ presentan potenciales de corrosión similares cuando son obtenidos a partir del mismo proceso de molienda, independientemente del contenido y tipo de refuerzo añadido.

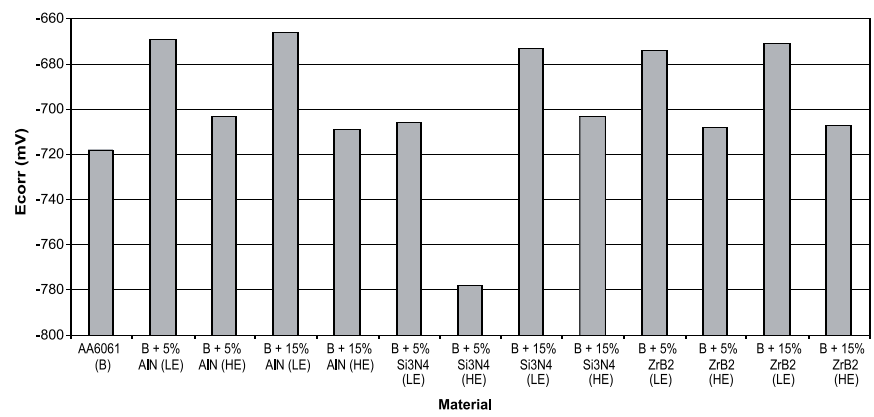

Figura 2.- Potencial de Corrosión de la aleación AA6061 y de los materiales compuestos a las 6 horas de ensayo. Ensayo de inmersión no normalizado (siete días en la disolución 3.5\% $\mathrm{NaCl}$ en 11 de $\mathrm{H}_{2} \mathrm{O}$ destilada). 
De este modo, los materiales presentan valores del potencial de corrosión y, en consecuencia, un comportamiento similar en función del tipo de molienda empleado en su proceso de fabricación ya sea baja o alta energía.

Este comportamiento puede ser índice de la influencia del método de molienda (a alta o baja energía) en el comportamiento frente a la corrosión de los materiales, en comparación con el papel que juegan otros factores como el tipo y contenido de refuerzo empleado.

Comparando los resultados obtenidos a partir de ambos ensayos, normalizado y no normalizado, y representados en las figura 3 , se puede observar en primer lugar la susceptibilidad que presentan todos los materiales al medio de corrosión. Sin embargo, al igual que se ha venido observando, comparando los resultados obtenidos por ambos ensayos, la tendencia que presentan es la misma: los materiales procesados por molienda de alta energía presentan un potencial de corrosión mayor.

\subsection{Estudio del potencial de picadura}

En la figura 4 aparece representadas, a modo de ejemplo, las curvas de polarización cíclica correspondientes a dos de los materiales estudiados.

Todos los materiales estudiados presentaron curvas de polarización similares. El potencial de repasivación de todos ellos ha sido

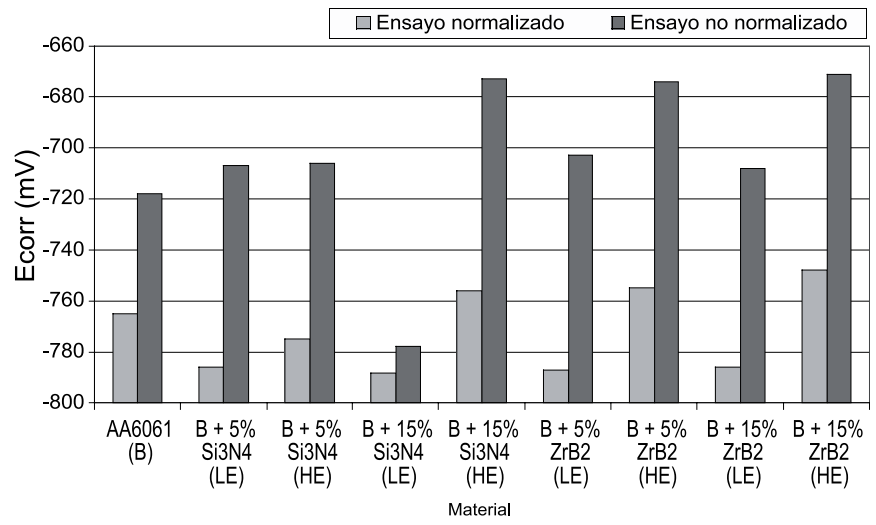

Figura 3. Potencial de Corrosión de la aleación AA6061 y de los materiales compuestos. Ensayo de inmersión normalizado y no normalizado.
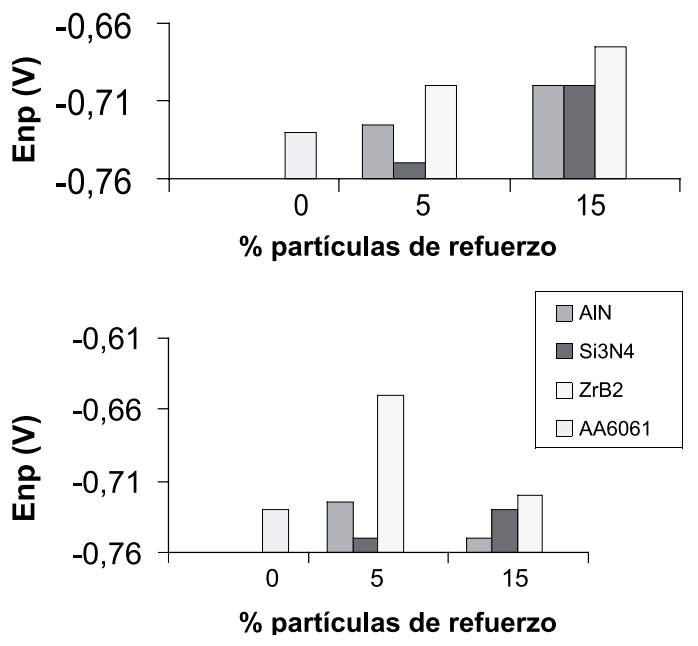

Figura 5 .- Potenciales de picadura de los materiales ensayados según porcentaje de refuerzo y tipo de molienda mayor que el potencial de corrosión, lo que indica la capacidad de todos ellos a la regeneración de la capa protectora frente a eventuales roturas de la misma.

Si se representan los potenciales de picadura obtenidos a partir de las curvas de polarización cíclica para los distintos materiales compuestos, figura 5, se puede observar que los materiales procesados por molienda de baja energía presentan mayores valores cuando se emplea el mayor de los porcentajes en refuerzo. Sin embargo, esta tendencia no se observa cuando se emplea la molienda por alta energía, posiblemente debido al menor tamaño del refuerzo que se fractura durante la molienda y se incorpora a la matriz con un tamaño menor.

\subsection{Estudio del potencial de repasivación}

En la representación de los potenciales de repasivación obtenidos a partir de las curvas de polarización cíclica para los distintos materiales compuestos, Figura 6, se puede observar que los materiales con un $15 \%$ de refuerzo y molienda de alta energía presentan los valores más altos. También se puede observar la poca o nula influencia del tipo de refuerzo cuando se añade un 5\% del mismo, tanto a alta como a baja molienda. Esto puede indicar que en los materiales reforzados estudiados no tiene un papel significativo ni los porcentajes de refuerzo utilizados ni el tipo de molienda empleado sobre el potencial de repasivación.

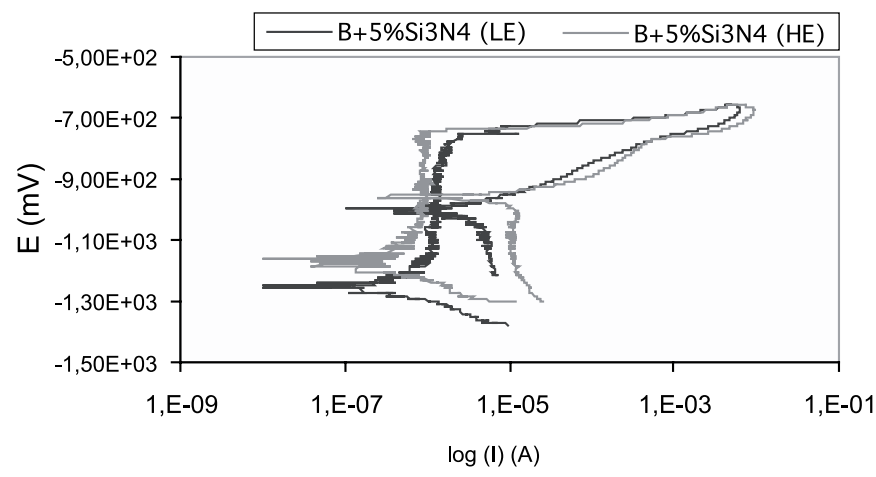

Figura 4. Curvas de polarización cíclica de los materiales compuestos reforzados con $5 \%$ de $\mathrm{Si}_{3} \mathrm{~N}_{4}$ por alta y baja energía.

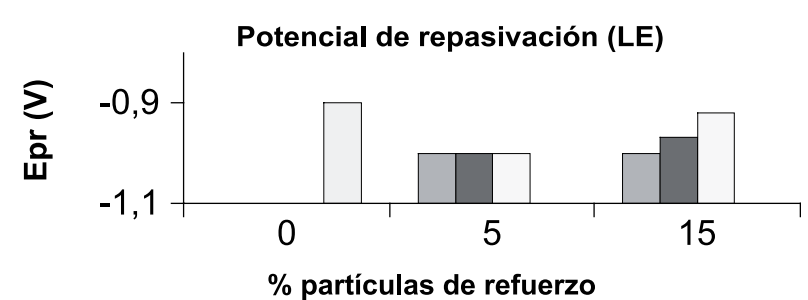

Potencial de repasivación (LE)

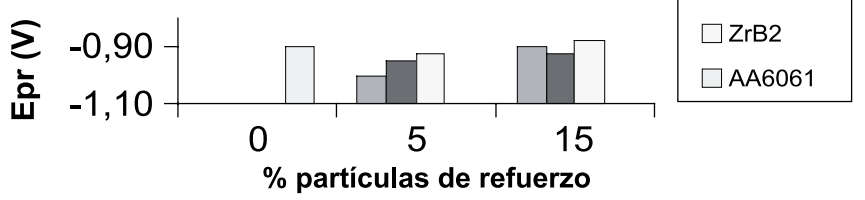

Figura 6. Potenciales de repasivación de los materiales ensayados según porcentaje de refuerzo y tipo de molienda 


\section{CONCLUSIONES}

Las conclusiones más significativas de este trabajo han sido las siguientes:

- Los resultados obtenidos a partir de ambos ensayos normalizado y no normalizado para la evaluación del potencial de corrosión presentan la susceptibilidad que presentan todos los materiales al medio de corrosión en función del tipo de refuerzo y del tipo de molienda.

- Los resultados obtenidos en ambos ensayos se sigue la misma tendencia: todos los materiales procesados por molienda de alta energía presentan potenciales de corrosión mayores, posiblemente debido al menor tamaño del refuerzo que se fractura durante la molienda y se incorpora a la matriz con un tamaño menor.

- Los materiales presentaron curvas de polarización similares, con zonas pasivas, potenciales de picadura y potenciales de repasivación claramente diferenciables. De ellas se pueden destacar los siguientes resultados: los materiales procesados por molienda de baja energía presentan mayores valores de potencial de picadura cuando se emplea porcentajes de refuerzo mayores. Finalmente, el papel que juega tanto el contenido en refuerzo como el tipo de molienda empleado sobre el potencial de repasivación no es significativo.

\section{BIBLIOGRAFÍA}

1. D.P. Bishop et al., M.S. and Engineering A. 290 (1-2) 16-24 (2000)

2. S.V. Prasad y P.K. Rohatgi, Journal of Metals, 22-26 (1987).

3. J. Eliasson y R. Dandström. $4^{\text {th }}$ International Conference on Aluminium Alloys, 535-542 (1994)

4. M. Hull. Powder Metallurgy, 40, 102-103 (1997).

5. J.B. Fogagnolo, E.M. Ruiz-Navas, M.H. Robert, J.M. Torralba, Scripta Materialia, 47(4), 243-248 (2002)

6. E.M. Ruiz-Navas, F. Velasco, C.E. da Costa, J.B. Fogagnolo, J.M. Torralba, Proceedings del VI Congreso Nacional de Mecânica Aplicada e Computacional, 1, 423-430 (1999)

7. J.B. Fogagnolo, E.M. Ruiz-Navas, M.H. Robert, F. Velasco, J.M. Torralba, Proceedings of Second Internacional Conference on Powder Metallurgy Science, 1, 21-27 (2000).

8. J.M. G. de Salazar, A. Ureña, S. Manzanedo, M.I. Barrena, Corrosion Sciencie, 41(3), 529-545 (1999).

Recibido: 1.2 .03

Aceptado: 30.11 .03 